\title{
Treatment of Sigmoid Volvulus by Deflation Versus Surgery: A Single Site Report
}

\author{
Yonas Yilma Raru ${ }^{1}$, Patricia R Messmer ${ }^{2}$, Arthur R Williams $^{3}$, Dawood H Sultan ${ }^{4}$, Ashenafi Berhanu Adale ${ }^{5}$, Yalelet Fentaw Shiferaw ${ }^{6}$, Paul \\ Hart $^{7}$ and Hugh Pettigrew ${ }^{7}$
}

${ }^{1}$ Assistant Professor of Surgery and Medical Director of Gondar University Hospital, Gondar, Ethiopia

${ }^{2}$ Consultant for Nursing Research \& Education, Benjamín León School of Nursing, Miami Dade College, Miami, Florida, USA

${ }^{3}$ Research Associate, CINDRR, James A. Haley VA Medical Center, Tampa, Florida, USA, and Visiting Professor, George Mason University, Fairfax, Virginia, USA ${ }^{4}$ Director, Centre for Education and Youth Development, Peercorps Trust Fund, Dar Es Salaam, Tanzania and Executive Director, Investigaciones Traslacionales, Soluciones Logísticas y Estratégicas de Panamá, Panama City, Republic of Panama

${ }^{5}$ Chief Resident, Gondar University Hospital, Gondar, Ethiopia

${ }^{6}$ Inpatient Coordinator and HMIS Director, Gondar University Hospital, Gondar, Ethiopia

${ }^{7}$ Consultants to the Medical School, University of Gondar, Gondar, Ethiopia

\begin{abstract}
Background: Although very common in developing regions like Africa, sigmoid volvulus (SV) has a much lower incidence in the West. There is a dearth of literature on the treatment of SV in countries with limited resources. This paper reports study results using a cohort of 200 SV patients in a low-income developing country.

Objectives: The aim of this study was to identify the characteristics associated with length of hospital stay (LOS) in the largest sample of sigmoid volvulus patients drawn from a single site within a single year and to examine the effects of treatment complications and previous attacks of SV on LOS.

Methods: Data from charts for a retrospective sample of 200 adult SV patients hospitalized in 2012 at Gondar University Hospital, Ethiopia, were statistically analyzed to determine LOS by patient characteristics and the effects of complications on LOS.

Results: Among the 200 patients diagnosed with SV, the ratio of deflation to surgery was almost 2:1 ratio to surgery. The total patient days in the study were 856 days. Patients with LOS $>5$ days accounted for $79 \%$ of patient days. Patients with a maximum LOS of 30 days accounted for $10.5 \%$ of total patient days. The shortest LOS (1.36 days) was among patients who underwent sigmoidoscopic decompression only and had no treatment complications. Complications associated with surgery accounted for 10.6 to 17.0 LOS days. Age, gender, gangrene and previous attacks were not significantly associated with surgery. Among the study patients, there were only five cases of failed deflation followed by surgery. All but five of the patients with gangrene had surgery. Shock, stroke, abscess, sepsis and surgery significantly prolonged LOS. Patients $(66 \%)$ had not experienced previous attacks. SV affected middle aged men more than the elderly men. Females had an excess relative risk $(R R=1.62)$ for surgery but was not statistically significant due to the small number of females $(n=15)$ in the study.
\end{abstract}

Conclusion: Deflation was an effective treatment modality, while surgery was performed only when indicated and only on the critical cases. Patients who are treated with deflation may avoid surgery which can be associated with additional complications. Avoidance of surgery and complications can substantially reduce LOS with subsequent and system costs.

Keywords: Sigmoid volvulus; Ethiopia; Africa; Surgery

\section{Introduction}

Volvulus is a twisting of the intestine on itself and its mesentery, causing an intestinal obstruction. It occurs most frequently in men and usually involves the sigmoid colon [1]. Sigmoid volvulus (SV) has variable and racial distributions. Although extremely common in developing societies like Africa, where it affects primarily young adult males, SV has a much lower incidence in the West, where it affects older and frail females. While chronic constipation is blamed for SV in the West, a high fiber diet is thought to be a major risk factor among African populations. Vergase [2] states that Ethiopians have long and mobile colons in addition to diet predisposing to volvulus. A large sigmoid colon distended with gas caused by a high-fiber diet is more likely to twist on its mesentery, which is the commonest cause of large gut obstruction in Africans [3]. Disease management involves relief of obstruction and the prevention of recurrent attacks $[4,5]$. There is a dearth of literature on the treatment of SV in countries with limited resources. The higher incidence and cost of treatment of SV in developing regions adds to pressure on scarce resources available for medical treatment. Significant contributions to health care costs are extended length of stay (LOS) and complications arising from treatments that lead to additional resource consumption. An important concern is the complication of gangrene during treatments for SV. This paper focuses on factors associated with both LOS and the appearance of gangrene.

\section{Study Objectives}

The aim of this study was to identify the characteristics associated with length of hospital stay (LOS) and to examine the effects of

*Corresponding author: Messmer PR, PhD, RN-BC, FAAN, 2Consultant fo Nursing Research \& Education, Benjamín León School of Nursing, Miami Dade College, Miami, Florida, USA, Tel: 954-309-1034; E-mail: pmessmer@mdc.edu

Received July 23, 2015; Accepted September 07, 2015; Published September 14, 2015

Citation: Raru YY, Messmer PR, Williams AR, Sultan DH, Adale AB, et al. (2015) Treatment of Sigmoid Volvulus by Deflation Versus Surgery: A Single Site Report. Surgery Curr Res 5: 247. doi:10.4172/2161-1076.1000247

Copyright: ( 2015 Raru YY, et al. This is an open-access article distributed under the terms of the Creative Commons Attribution License, which permits unrestricted use, distribution, and reproduction in any medium, provided the original author and source are credited. 
treatment complications and previous attacks of SV on LOS. In this study, it was hypothesized that deflation, a conservative approach to SV, can be effective in resource-poor regions with surgery recommended for the more critical cases. This study reports on the largest sample of patients who were assessed for SV drawn from a single site within a single year.

The review of the literature below suggests that many factors are relevant to the effective treatment of SV. Some of these are outlined in the findings and discussion section of this paper. The unusual data set used in this study and the presentation in this paper addresses some important considerations related to LOS and the appearance and treatment of gangrene. However, the relative rarity of some conditions and the difficulty of collecting accurate, extensive information in developing regions limits what can be done with patient level data.

\section{Review of the Literature}

The articles reviewed herein are largely from low-income nonWestern developing countries, since SV appears to manifest itself differently between Western and non-Western cultures. While chronic constipation is blamed for the Western type of SV, a high fiber diet and particular physiologic features are considered a major risk factor for SV among African populations. For instance, Vergase [2] noted that Ethiopians have long, mobile colons and typically consume high-fiber diet; both variables are predisposing to SV. A large sigmoid colon distended with gas caused by a high-fiber diet is more likely to twist on its mesentery, which is the commonest cause of large gut obstruction in Africans [3].

A search through Medline, PubMed, Cinahl, and Ovid databases revealed that only16 reported SV studies were conducted in nonWestern settings [6-20,25]. Only one of these studies examined lengths of stay (LOS) or sequelae associated with LOS and treatment. The rest of the studies usually reported some patient demographics and selected conditions associated with treatment. Several studies concluded that deflation is a safe method to use with patients, but these appear to be uncontrolled observational studies based on data from small numbers of patients. Most of the papers commented upon gangrene and effects of gangrene on treatment outcomes.

Mnguni et. al, [6] reported that SV predominately affected young African males and found that the timing of surgery, the type of anastomosis and the viability of the bowel did not influence surgical outcomes in 135 patients ( 122 males; with a mean age of 39.3 years; SD \pm 17 years). Complications and mortality occurred in $14 \%$ and $17 \%$ of the study patients, respectively.

Tan, Chong and Sim [7] who studied 71 patients in a Singapore hospital over approximately nine years reported that $90.1 \%$ of SV patients were initially treated conservatively using a flatus tube and/ or sigmoidoscopic decompression. Emergency surgery was associated with a mortality rate of $17.6 \%$. So, elective surgery was suggested in view of the high recurrence rate $(>60 \%)$ and the considerable risks of emergency surgery. Another retrospective study by Ghariani, Houissa and Sebai [8] on 40 adult patients treated at a Tunisian hospital indicated that the best treatment consisted of an endoscopic volvulus removal followed by a sigmoidal resection during the same hospitalization period.

Lou et al., [9] reported that colonoscopy decompression and de-rotation was the primary emergency treatment of choice in 28 uncomplicated acute SV patients in Shanghai Hospital, Shanghai, China over a 12 year period (January, 2001-July, 2012). Emergency surgery was reserved for patients with whom non-operative treatment was unsuccessful or in patients with peritonitis. Furthermore, Khan et al., [10] reported that percutaneous endoscopy colostomy (PEC) was an effective alternative in 8 of 12 high risk elderly patients in the United Kingdom treated for recurrent SV. Although PEC prevented colonic resection in 7 of the 8 patients, one patient died from postoperative complications after sigmoid resection.

Furthermore, after reviewing 39 of 57 patients from New Zealand who ultimately had surgery over a 20 year period, Yassaie, ThompsonFawcett and Rossaak [11] suggested that early elective surgery for SV was encouraged when prohibitive co-morbidities were not present. After reviewing 39 of 57 patients who ultimately had surgery over a 20 year period (January 1989 - January 2009), early elective surgery was encouraged in patients without prohibitive co-morbidities. Traore and colleagues [12] conducted a retrospective study (1996-2010) of 417 patients in Bamako, Mal and found that the surgical approach had an impact on the mortality of patients who were in poor general condition.

Onder et al. [13] studied 158 adults [135 men (85.4\%) and 23 women $(14.8 \%)$ with an overall mean age of 62.54 years] Turkish SV patients over a 16 year period. They found that surgery-related complications were more frequent and were associated with the risk of mortality. The study found that surgery-related complications, such as wound infection and intra-abdominal abscess were more frequent in surgical patients and were associated with the risk of mortality. The study also found that delayed admission resulted in higher risks of mortality. These risks were reduced through early patient admission, intensive preoperative resuscitation, administration of appropriate antibiotics, and performance of emergency and viable surgery.

And, Atamanalp [14] who studied 952 Turkish patients at another hospital over a period of 46.5 years found that among 686 patients who underwent nonsurgical detorsion the success rate was $77.1 \%$, and that the rates for comorbidity, mortality and early recurrence were $2.5 \%, 0.7 \%$ and $4.5 \%$ respectively. Emergency surgeries were performed in 447 patients with $35.5 \%$ comorbidity, $16.1 \%$ mortality, and $0.7 \%$ early recurrence rates while elective surgical treatment was performed in 104 patients with $12.5 \%$ comorbidity, no mortality and no recurrence. Atamanalp et al., [15] also studied 442 patients from the same data set. They found that $271(61.3 \%)$ patients had sigmoid gangrene. Though they found that the presence of pregnancy was negatively correlated with gangrene development, they determined that comorbid diseases associated with shock, prolonged symptom duration, over rotation, and associated ileo-sigmoid knotting had significant positive correlations with bowel gangrene.

Furthermore, Bekele et al., [16] found that gangrenous SV in 56 (36.1\%) patients necessitated a colostomy. Also to be noted is the finding by Sozen and colleagues [17] that the mean LOS, wound infection, and mortality rates did not differ significantly between blow-hole colostomy and the Hartmann's procedure groups. However superficial wound infection was higher in the blow-hole colostomy group (32\% vs. $15 \%$ ). In addition, Kassi and colleagues [18] who studied 25 patients who underwent the Hartmann's procedure in Côte d'Ivoire over a 10 year period found that the procedure was associated with significant mortality. Another study [19] reviewing 42 Turkish patients who underwent primary resection and anastomosis or Hartmann's procedure indicated that sigmoidectomy was an effective procedural option.

Also, in a study of 41 Iraqi patients randomized into two groups to compare percutaneous deflation prior to emergency tube decompression 
followed electively by colopexy with banding versus emergency tube decompression followed electively by sigmoid colectomy, Salim [20] found that percutaneous deflation was both a safe and fast method for successful sigmoidoscopic decompression and emergency surgery avoidance. The study concluded that colopexy by branding was a more advantageous elective surgical procedure than mesenteropexy and resectional surgery. Other studies indicated that laparoscopic resection for decompressed SV was a useful alternative in high risk patients or in the elderly who may not tolerate conventional colonic surgery [21,22].

In this study, it was hypothesized that deflation is effective in resource poor regions, with expensive emergency surgery recommended only for critical cases. Effectively deflated patients who needed elective resection of redundant sigmoid to prevent recurrence of the volvulus were more likely to avoid emergency surgery which might lead to complications. The aims of this study, therefore, were to identify the frequency, length of hospital stay (LOS), and the number of gangrenous bowels in an Ethiopian population using the largest sample of patients who were assessed for sigmoid volvulus over a single year.

In contrast, the studies we reviewed used either small samples obtained during a limited time period or large samples obtained over decades. Problems of studies with small samples are well known. However, studies that use observations collected over long periods of time are also problematic, especially when medical procedures, facilities, and clinical skills may have changed dramatically. Hence, the nature of data used in this study makes it unique and timely.

\section{Methods}

\section{Setting}

Gondar University Hospital (GUH) is the second largest hospital in Ethiopia. It serves about six million people spread over a $100 \mathrm{~km}$ radius. It has approximately 500 beds in six departments and 15 wards with a wide range of specialty services. Nearly 150,000 outpatient visits and more than 14,000 hospital admissions are recorded at GUH every year. Approximately 6,000 surgeries and 2,000 deliveries are attended annually. Services provided include prevention, care and support of HIV/AIDS, TB, and related problems especially multidrug resistance TB. The Kalazar project for Visceral Leishmaniasis has its own clinic with 24 beds for new drug trials and patient treatment. As a teaching hospital, GUH is involved in producing competent health professionals and many of the physicians are engaged in research activities. It boasts over 75 doctors, 280 nurses, 20 pharmacists and 35 laboratory technicians who have roles in teaching medical and nursing students.

\section{Study sample}

A retrospective sample of adult (age $\geq 18$ years) patients admitted at GUH between January 1 and December 31, 2012 was used. Charts for 487 patients diagnosed with sigmoid volvulus were reviewed by a team of four surgeons, a nurse researcher and the nurse Inpatient Coordinator. Of these patients, 200 cases were treated by the same four surgeons. These surgeons followed identical treatment protocols. Their patients constituted the study sample. Information extraction from patient charts was exempted from review by the Ethics Board at GUH.

\section{Variables}

In this study, patient age was measured in years and length of hospital stay (LOS) in days. Gender (male, female), treatment type (deflation, surgery) and treatment complication categories (list) and gangrene were measured in binary $(0,1)$ terms. Previous SV attacks were measured on a 1-5 scale reflecting the number of previous attacks.
In the statistical analysis, the LOS for patients who had less than one full day of stay at hospital was assigned a value of 1.00 .

\section{Statistical analysis}

Frequency distributions, ordinary least-squares (OLS) regressions, and logistic regressions were used to analyze the data. Data analysis was conducted using Stata version 13.0 [23]. The statistician analyzing the data did not treat patients nor had he previously been involved with the physicians or research team.

\section{Results}

Table 1 provides demographic and clinical data pertaining to the 200 study patients. It shows that the ratio of deflation to surgery was almost 2:1. Almost all study patients were male (92.5\%). The mean LOS was 4.30 days ( $S D=5.59$ days). The majority of patients $(66.5 \%)$ had a LOS of less than 5 days, $33.5 \%$ had a LOS equal to or greater than 5 days, $25.5 \%$ developed SV-associated gangrene and only $16 \%$ experienced post-surgical complications: $2.0 \%$ had stroke, $2.5 \%$ experienced deflation failure, $2.0 \%$ developed intra-abdominal abscess, $7.0 \%$ developed sepsis, and $2.5 \%$ had septic shock. The majority (66\%) of patients did not report a previous SV attack. Of those who reported an attack (68 patients), $57.4 \%$ had a single attack, $32.4 \%$ had two, $8.8 \%$ had three, and only $1.5 \%$ (one patient) reported five attacks.

Logistic regressions indicate that female patients have an excess risk of $62 \%$ for surgery (the point estimate of relative risk $[R R]=1.62$ ). However, difference in RR by gender was not significant $(p=0.11)$. Of the five study patients who died, all except one died within 10-25 days. One patient died in the first post-surgery day. There were no other deaths among the patients after a year.

Table 2 shows the distribution of a total 856 LOS days. Patients with LOS in excess of 5 days accounted for $79 \%$ of the total days in this sample. Patients with a maximum LOS of 30 days accounted for $10.5 \%$ of the days in the sample.

Table 3 displays regression model estimates of net contributions of each complication to LOS. The model has a significant fit to the data and accounted for $53 \%$ of the variance in LOS. The effects of age, gender, other complications (gangrene), and previous attacks were not significant and were, therefore, not reported. Estimated coefficients indicate that shock; stroke, abscess, sepsis and surgery significantly prolonged LOS beyond the 1.36 days (intercept) which patients spent in hospital if they underwent sigmoidoscopic decompression only and had no complications. Net contributions of each complication to LOS ranged from 3 days (sepsis $=1.36+2.42)$ to 10 days (abscess $=1.36+$ $8.85)$. Surgery added 6.79 days to any treatment.

Table 4 displays estimates of LOS among SV patients by treatment status. The shortest LOS (1.36 days) was experienced by patients who only underwent sigmoidoscopic decompression and had no treatment complications. Complications associated with surgery accounted for 10.6 to 17.0 LOS days.

The majority of the patients (66\%) did not experience SV attacks prior to presentation at clinic. Only 68 patients had one or more previous attacks. Of these, more than half $(57.4 \%)$ reported a single previous attack (Table 5).

Overall, estimates indicate a significant negative correlation $(\mathrm{r}=-$ $0.2787, \mathrm{p}<.0001$ ) between previous attacks and gangrene. That is, the more previous attacks the less likely the patient was to have gangrene. But, when the gangrene correlation was restricted only to the 68 patients 
Citation: Raru YY, Messmer PR, Williams AR, Sultan DH, Adale AB, et al. (2015) Treatment of Sigmoid Volvulus by Deflation Versus Surgery: A Single Site Report. Surgery Curr Res 5: 247. doi:10.4172/2161-1076.1000247

Page 4 of 6

\begin{tabular}{|c|c|c|}
\hline Variables & Categories & Results, $\mathrm{N}$ of Subjects and \% \\
\hline Age & Age Range $19-85$ years & Mean 53.69 years $(S D=15)$ \\
\hline Gender & $\begin{array}{l}\text { Males } \\
\text { Females }\end{array}$ & $\begin{array}{c}185(92.5 \%) \\
15(7.5 \%)\end{array}$ \\
\hline Treatment & $\begin{array}{l}\text { Deflation } \\
\text { Male } \\
\text { Female } \\
\text { Surgery } \\
\text { Male } \\
\text { Female }\end{array}$ & $\begin{array}{r}131(65.5 \%) \\
124(62 \%) \\
7(38 \%) \\
69(34.5 \%) \\
61(31 \%) \\
8(4 \%)\end{array}$ \\
\hline LOS Central Tendencies & All Subjects & $\begin{array}{l}\text { Mean } 4.28 \text { days }(\mathrm{SD}=5.59) \\
\text { Mode/Median } 1.00 \text { day }\end{array}$ \\
\hline LOS by Groupings & $\begin{array}{l}\qquad \begin{array}{l}\text { LOS }<\text { than } 5 \text { days } \\
\text { LOS } \geq 5 \text { days }\end{array} \\
\text { LOS } \leq 5 \text { days with complications } \\
\text { Only } 5 \text { patients with complications and LOS }>5 \text { days }(2.5 \% \text { of all } \\
\text { patients) }\end{array}$ & $\begin{array}{c}133(66.5 \%) \\
67(33.5 \%) \\
27(13.5 \% \text { of all patients })\end{array}$ \\
\hline Gangrene & $\begin{array}{l}\text { Yes } \\
\text { No }\end{array}$ & $\begin{array}{r}51(25.5 \%) \\
145(74.5 \%)\end{array}$ \\
\hline Complications & $\begin{array}{l}\text { No complications } \\
\text { Stroke } \\
\text { Deflation failure } \\
\text { Intra-abdominal abscess } \\
\text { Sepsis } \\
\text { Septic shock }\end{array}$ & $\begin{array}{rr}168 & (84.0 \%) \\
4 & (2.0 \%) \\
5 & (2.5 \%) \\
4 & (2.0 \%) \\
14 & (7.0 \%) \\
5 & (2.5 \%)\end{array}$ \\
\hline \multirow[t]{7}{*}{ Previous attacks } & No Previous attacks & $168(66 \%)$ \\
\hline & Previous attacks & $68(34 \%)$ \\
\hline & One & $39(57.4 \%)$ \\
\hline & Two & $22(32.4 \%)$ \\
\hline & Three & $6(8.8 \%)$ \\
\hline & Four & $0(0.0 \%)$ \\
\hline & Five & $1(1.5 \%)^{\star}$ \\
\hline
\end{tabular}

Percentage figures add $100.1 \%$ due to rounding up.

Table 1: 200 Cases of Sigmoid Volvulus at a Single Site with the Same Attending Physician.

\begin{tabular}{|c|c|c|c|}
\hline Length of Stay & Freq. & Percent & Cum.percentage \\
\hline One day & 116 & 58.00 & 58.00 \\
\hline Two days & 15 & 7.50 & 65.50 \\
\hline Three days & 2 & 1.00 & 66.50 \\
\hline Five days & 6 & 3.00 & 69.50 \\
\hline Six days & 8 & 4.00 & 73.50 \\
\hline Seven days & 11 & 5.50 & 79.00 \\
\hline Eight days & 11 & 5.50 & 84.50 \\
\hline Nine days & 6 & 3.00 & 87.50 \\
\hline Ten days & 3 & 1.50 & 89.00 \\
\hline Eleven days & 2 & 1.00 & 90.00 \\
\hline Twelve days & 3 & 1.50 & 91.50 \\
\hline Thirteen days & 1 & 0.50 & 92.00 \\
\hline Fourteen days & 4 & 2.00 & 94.00 \\
\hline Fifteen days & 2 & 1.00 & 95.00 \\
\hline Sixteen days & 2 & 1.00 & 96.00 \\
\hline Seventeen days & 1 & 0.50 & 96.50 \\
\hline Eighteen days & 1 & 0.50 & 97.00 \\
\hline Twenty days & 2 & 1.00 & 98.00 \\
\hline Twenty-three days & 1 & 0.50 & 98.50 \\
\hline Thirty days & 3 & 1.50 & 100.00 \\
\hline Total & 200 & 100.00 & \\
\hline
\end{tabular}

Table 2: Distribution of LOS among the Study Sample.

with one or more previous attacks, the direction of the association changed (Table 6). It became positive but was statistically not significant $(\mathrm{r}=0.1790, \mathrm{p}=0.2944)$. Table 6 shows why this relationship was not significant. Only five of the 68 patients with previous attacks in the sample had gangrene. Four of these subjects had gangrene reported during the first previous attack. One subject had gangrene, but not during the first previous attack. Fifty-one patients had gangrene with one or more previous attacks while 46 patients had gangrene with no 


\begin{tabular}{|c|c|c|c|c|}
\hline Variable & Coefficient & $\mathbf{P}$ & Confidence Interval & LOS for Direct Effects (days) \\
\hline Shock & 5.01 & 0.005 & $(1.53,8.49)$ & 6.37 \\
\hline Stroke & 5.45 & 0.005 & $(1.64,9.25)$ & 6.81 \\
\hline Abscess & 8.85 & 0.000 & $(4.93,12.77)$ & 10.21 \\
\hline Sepsis & 2.42 & 0.038 & $(0.13,4.71)$ & 3.78 \\
\hline Surgery & 6.79 & 0.000 & $(5.52,8.06)$ & 8.15 \\
\hline Intercept & 1.36 & 0.000 & $(0.69, \quad 2.02)$ & 1.36 \\
\hline
\end{tabular}

$R^{2}=0.55$ Adj $R=0.53 ; P<0.0001 ; N=199$

Table 3: Effects of complications on LOS among patients diagnosed with Sigmoid Volvulus in an Ethiopian hospital in 2012.

\begin{tabular}{|c|c|}
\hline Treatment Status & LOS (in days) \\
\hline No complications & 1.36 \\
\hline Surgery and no complications & 8.15 \\
\hline Surgery and Shock & 13.16 \\
\hline Surgery and Stroke & 13.60 \\
\hline Surgery and Abscess & 17.00 \\
\hline Surgery and Sepsis & 10.57 \\
\hline
\end{tabular}

$P \leq 0.05$ indicates statistically significant effects

Table 4: Estimates of LOS among Ethiopian Sigmoid Volvulus patients with or without treatment complications, 2012

\begin{tabular}{|c|c|c|}
\hline Previous attack & Frequency. & Percentage \\
\hline 0 & 132 & 39 \\
\hline 1 & 22 & 66.00 \\
\hline 2 & 6 & 19.50 \\
\hline 3 & 1 & 3.00 \\
\hline 5 & $\mathbf{2 0 0}$ & 0.50 \\
\hline Total & $\mathbf{1 0 0 . 0 0}$ \\
\hline
\end{tabular}

Table 5: Previous Attacks of Sigmoid Volvulus in Ethiopian patients.

\begin{tabular}{|l|c|c|c|c|c|}
\hline Gangrene & \multicolumn{3}{|c|}{ Previous Attacks } & \multicolumn{2}{|c|}{ Total } \\
\hline Number of attacks & 0 & 1 & 2 & 5 \\
\hline 0 & 86 & 35 & 21 & 6 & 1 \\
\hline 1 & 46 & 4 & 1 & 0 & 0 \\
\hline Total & $\mathbf{1 3 2}$ & $\mathbf{3 9}$ & $\mathbf{6}$ & $\mathbf{2 2}$ \\
\hline
\end{tabular}

Odds Ratio $(\mathrm{OR})=0.1484 ; 95 \%$ Confidence Interval $(\mathrm{Cl})=0.0439,0.4053$ Chi-square $=17.86 ; \mathrm{p}<0.0001$

Table 6: Gangrene associated with previous attacks.

previous attacks. In contrast, 63 patients had previous attacks but no gangrene $(\mathrm{OR}=0.1484$, Chi square $=17.86, \mathrm{p}<0.0001)$. That is, a previous attack may have been a "protective factor" reducing the risk of having gangrene in this cohort.

\section{Discussion}

This paper examined previously unstudied factors associated with both LOS and the appearance of gangrene among Ethiopian patients. The findings of this study indicate that compared to surgery, deflation is an effective treatment for sigmoid volvulus for many patients. Among the study patients, there were only five cases of failed deflation followed by surgery. Deflation also significantly contributed to reductions in LOS. Following identical treatment protocols enabled the surgeons to avoid surgery and reduce treatment complications. Surgery avoidance and control of complications substantially reduced LOS. A shorter LOS in hospital is an important, but largely unstudied, issue in resource poor African settings where it would directly reduce system and subsequent costs.

Two thirds of the patients in this study underwent deflation with emergency surgery recommended only for critical cases. The overwhelming majority (84\%) of patients reported no complications following deflation. Treatment success may have been the result of the protocols followed at the study site [24]. Because many patients with a first attack will develop gangrene relative to those with repeated attacks, these patients were deflated under sigmoidoscopy guidance. During the procedure, the surgeon also checked for viability of the bowel to determine whether or not it was a gangrenous sigmoid. The decision to perform deflation versus surgery depended upon the condition of the patient. When the surgeons had clinical suspicion (based on a secondary diagnosis of fever, tachycardia, low BP, or abdominal tenderness) of a gangrenous SV, patients were sent to surgery. The other possibility for choosing to perform surgery was when patients showed no sign of gangrene but the surgeons failed to successfully deflate them.

Patients were assessed individually and treatment decisions were made on a case by case basis. Patients who underwent surgery were mostly sicker because of a gangrenous bowel. Sometimes, however, very sick patients could have a viable sigmoid; and their very sick condition could be due to fluid and electrolyte disturbance. That was found in five patients who were labeled to have gangrenous sigmoid at clinical presentation, but after examination were not referred for surgery. 
Almost all patients who had previous attacks were treated with deflation. There were exceptions in cases of some patients with diagnoses of gangrenous sigmoid who were taken to the Operating Room for surgery and were found to have a viable sigmoid. The operating surgeons sometimes decided to only rotate the sigmoid rather than performing resection. If these patients returned with another SV attack, surgical deflation was done rather than sigmoidoscopic guided deflation.

After undergoing deflation, patients are at risk of recurrence of the volvulus if they did not have elective resection. Deflated patients were offered an elective surgery because of the known risk of recurrence. Although SV is an acute condition, the patients in this study who passed the early days of treatment appeared to have better survival rates than reported in the literature. The mortality rate among this group was $2.5 \%$. It was significantly lower than the $20 \%$ rate reported in a recent study of Ethiopian sigmoid volvulus patients treated at three teaching hospitals in Addis Ababa between July 2002 and June 2005 [25]. The high level of expertise of the four treating physicians and medical facilities ought to be considered when extrapolations are made to hospitals in other developing countries.

\section{Conclusions}

The majority of the patients did not experience SV attacks prior to presentation at this Ethiopian hospital. Among patients admitted for SV, deflation was an effective treatment modality. Surgery was done only on the more critical cases. The majority of patients had a LOS of less than 5 days with one-third having a LOS equal to or greater than 5 days. Surgery, shock and infectious complications increased the length of hospital stay of patients.

One quarter of the patients developed SV-associated gangrene and only $16 \%$ of all patients experienced post-surgical complications. However, patients who experienced repeated attacks of sigmoid volvulus appeared to have lower risks of developing gangrene.

Almost all of the study patients were male. Although the number of women included in this study was small, females may have an excess relative risk for surgery. This is, however, not unique to Ethiopian women [26].

Sigmoid volvulus affects more middle aged men in Gondar than older people. Patients who passed the early days of treatment had better survival rates (mortality rate $=2.5 \%$ ) than reported in the literature. Though evidence on quality of care and survival outcomes across Ethiopian hospitals is lacking, studies that examined these issues elsewhere suggest that the organizational culture, staffing, technology, use of protocols/checklists, and volume in teaching hospitals may lead to higher-quality care and survival advantages among patients [27].

This study used a large sample and is more current than most available studies. Its findings are likely to be of interest to physicians and researchers in gastroenterology, particularly those in resource constrained environments. However, the findings of this study are tempered by the fact that it relied on data from a single site. Also, factors influencing readmissions for previous attacks were not included in the current analysis, and, therefore, will need to be assessed in future prospective studies.

\section{References}

1. Price SA, Wilson LM (2003) Pathophysiology: Clinical Concepts of Disease Process. Mosby St Louis.
2. Vergase A (2009) Cutting for Stone 2009. Vintage Books New York, USA.

3. http://www.meb.uni-bonn.de/dtc/primsurg/docbook/html/x3450.html

4. Madidba TE, Thomson SR (2000) The management of sigmoid volvulus. JR Coll Surg Edinb 25: 74-80.

5. Sugimoto S, Mizukami T, Morohoshi Y, Komatsu H (2013) Sigmoid volvulus associated with Chilaiditis's syndrome. Intern Med 52: 515-516.

6. Mnguni, MN, Islam, J, Manzani, V, Govindasamy V, Zulu BM, et al. (2012) How far has the pendulum swung in the surgical management of sigmoid volvulus? Experience from the KwaZulu-Natal teaching hospitals and review of the literature. Colorectal Disease 14: 1531-1537.

7. Tan KK, Chong CS, Sim R (2010) Management acute sigmoid volvulus: an institutions' experience over 9 years. World J Surg 34: 1943-1948.

8. Ghariani B, Houissa H, Sebai F (2010) Management of sigmoid volvulus. Tunis Med 88: 163-167.

9. Lou Z, Yu ED, Zhang W, Meng RG, Hoa LQ, et al. (2014) Appropriate treatment of acute sigmoid volvulus in the emergency setting. World J Gastroenterology 19: $4979-4983$.

10. Khan MA, Ullah S, Beckly D, Oppong FC (2013) Percutaneous endoscopic colostomy (PEC): an effective alternative in high risk patients with recurrent sigmoid volvulus. J Coll Physicians Surg Pak 23: 806-809.

11. Yassaie O, Thompson-Fawcett M, Rossaak J (2013) Management of sigmoid volvulus: is early surgery justifiable? ANZ J Suerg 83: 74-78.

12. Traore D, Sanpgp ZZ, Bengaly B, Coulibaly B, Traore I, Goita D, et al. (2014) Acute sigmoid volvulus: results of surgical treatment in the teaching hospitals. J Visc Surg 151: 97-101.

13. Onder A, Kapan M, Arikanoglu Z, Palanci Y, Gumus M, et al. (2013) Sigmoid colon torsion: mortality and relevant risk factors. Eur Rev Med Pharmacol Sci 201317 Suppl 1: 127-132.

14. Atamanalp SS (2013) Treatment of sigmoid volvulus: a single-center experience of 952 patients over 46.5 years. Tech Coloproctiol 17: 561-569.

15. Atamanalp SS, Kisaoglu A, Ozogul B (2013) Factors affecting bowel gangrene development in patients with sigmoid volvulus. Ann Saudid Med 33: 144-148.

16. Bekele A, Kotisso B, Tesfaye M (2009) Patterns and indication of colostomies in Addis Abada, Ethopia. Africa Ethiop Med J 47: 285-290.

17. Sozen S, Das K, Erdem H, Menekse E, Cetinkunar S, et al. (2012) Resection and primary anastomosis with modified blow-hole colostomy or Hartmann's procedure. Which method should be performed for gangrenous sigmoid volvulus? Chirurgia (Bucur) 107: 751-755.

18. Kassi AB, Lebeau R, Yenon KS, Katche E, Diane B, et al. (2012) Morbidity and mortality of Hartmann's procedure for sigmoid volvulus at the Univrsity Hospital of Cocody, Abijan. West Afr J Med 30: 169-172.

19. Suleyman O, Kessaf AA, Ayhan KM (2012) Sigmoid volvulus; long tern surgical outcomes and review of the literature. S Afr J Surg 50: 9-15.

20. Salim AS (1990) Percutaneous deflation and colopexy for volvulus of the sigmoid; a new approach. JR Coll Surg Edinb 35: 356-359.

21. http://www.rcsed.ac.uk/RCSEDBack Issues/journal/vol45_2/4520003.htm

22. http://www.lapsurgery.com/volvulus.htm.

23. Stata Corp LP (2013) Stata version 13 for Windows: College Station, TX, USA

24. Gawande A (2009) The Checklist Manifesto. New York: Henry Holt and Company.

25. Kotisso B, Bekele A (2006) Ilio-sigmoid knotting in Addis Ababa: a three year comprehensive retrospective analysis. Ethiop Med J 44: 377-383.

26. http://www.meb.uni-bonn.de/dtc/primsurg/docbook/html/3450.htm

27. Ayanian JZ, Weissman JS (2002) Teaching Hospitals and Quality of Care: A Review of the Literature. Milbank Quarterly 80: 569-593. 